\title{
When tumefactive demyelination is truly a tumor: case report of a radiographic misdiagnosis
}

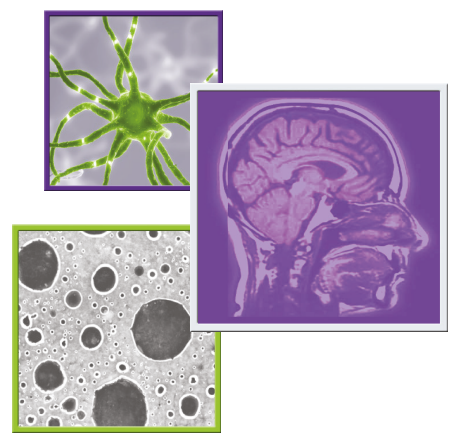

\author{
Breanna Taylor ${ }^{1}$, Mallika P Patel ${ }^{2} \&$ Katherine B Peters*,3 (iD) \\ ${ }^{1}$ Department of Pharmacy, University of Miami Health System, Sylvester Comprehensive Cancer Center, Miami, FL 33136, USA \\ ${ }^{2}$ Department of Pharmacy, Duke University Hospital, The Preston Robert Tisch Brain Tumor Center, Durham, NC 27710, USA \\ ${ }^{3}$ Department of Neurosurgery, Duke University School of Medicine, The Preston Robert Tisch Brain Tumor Center, Durham, NC \\ 27710, USA \\ *Author for correspondence: katherine.peters@duke.edu
}

Oligodendrogliomas are slow-growing tumors that account for $15-20 \%$ of gliomas. This case report describes the case of an adult male patient diagnosed initially with tumefactive demyelination and multiple sclerosis, which was subsequently found to be a well-differentiated low-grade oligodendroglioma. This case emphasizes the importance of timely diagnosis in oligodendrogliomas and other brain tumors for the prompt initiation of appropriate therapy, to minimize the likelihood of disease progression, ensure symptom management and escalation of unnecessary treatments for multiple sclerosis.

First draft submitted: 19 October 2020; Accepted for publication: 15 December 2020; Published online: 15 January 2021

Keywords: demyelination • multiple sclerosis $\bullet$ oligodendroglioma • tumefactive

Worldwide prevalence of multiple sclerosis (MS) is estimated to be roughly 2.8 million [1]. MS results in demyelination of the CNS and stems from an immune mediated attack on the white matter of the brain and spinal cord. Clinical manifestations may include sensory symptoms in the face and limbs, unilateral visual loss, acute motor weakness and gait disturbances. It is key to document dissemination in both space and time for a diagnosis of MS. MRI is the test of choice to support a diagnosis of MS and to evaluate the space and time elements required for an MS diagnosis [1]. MS lesions are traditionally found in the periventricular region, corpus callosum and centrum semiovale and the size of these lesions are usually below two centimeters in diameter [2]. In patients with MS, demyelinating lesions with mass effect can be referred to as tumefactive demyelination (TD). Other ancillary testing such as a lumbar puncture are not required for MS diagnosis but oligoclonal bands may be found in up to $95 \%$ of MS patients. Recent advancements in the treatment of MS have occurred with new disease modifying agents, however, not without significant toxicity associated with these medications [3,4]. Therefore, it is critical to provide the proper diagnosis of MS for patients when MRI findings are indicative of this condition. TD, previously believed to be very rare and representing only $1-3 / 1000$ cases, has in more recent studies found to represent a higher proportion of $1.4-8.2 \%$ of MS patients [5,6]. Tumefactive lesions are commonly described as mass-like lesions that are greater than two centimeters and that can exhibit a degree of enhancement, including ring enhancement [7-9]. The presence of tumefactive lesions on MRI may cause challenges in diagnosis and there have been reports of misdiagnosis $[10,11]$. In this case report, we present a patient that was initially diagnosed and treated for TD, but ultimately found to have low grade oligodendroglioma. Moreover, we highlight the MS related treatments that the patient received before diagnosis and treatment of his brain tumor.

\section{Case report}

A 31-year old previously healthy male, presented to the emergency department with a complex partial seizure. Prior to the emergency department visit, the patient was in a good state of health. Enhanced computed tomography revealed a possible subacute stroke or edema with an underlying mass in the left parieto-occipital area. Emergency department providers ordered MRI brain with and without contrast which revealed a large abnormal hyperintense 

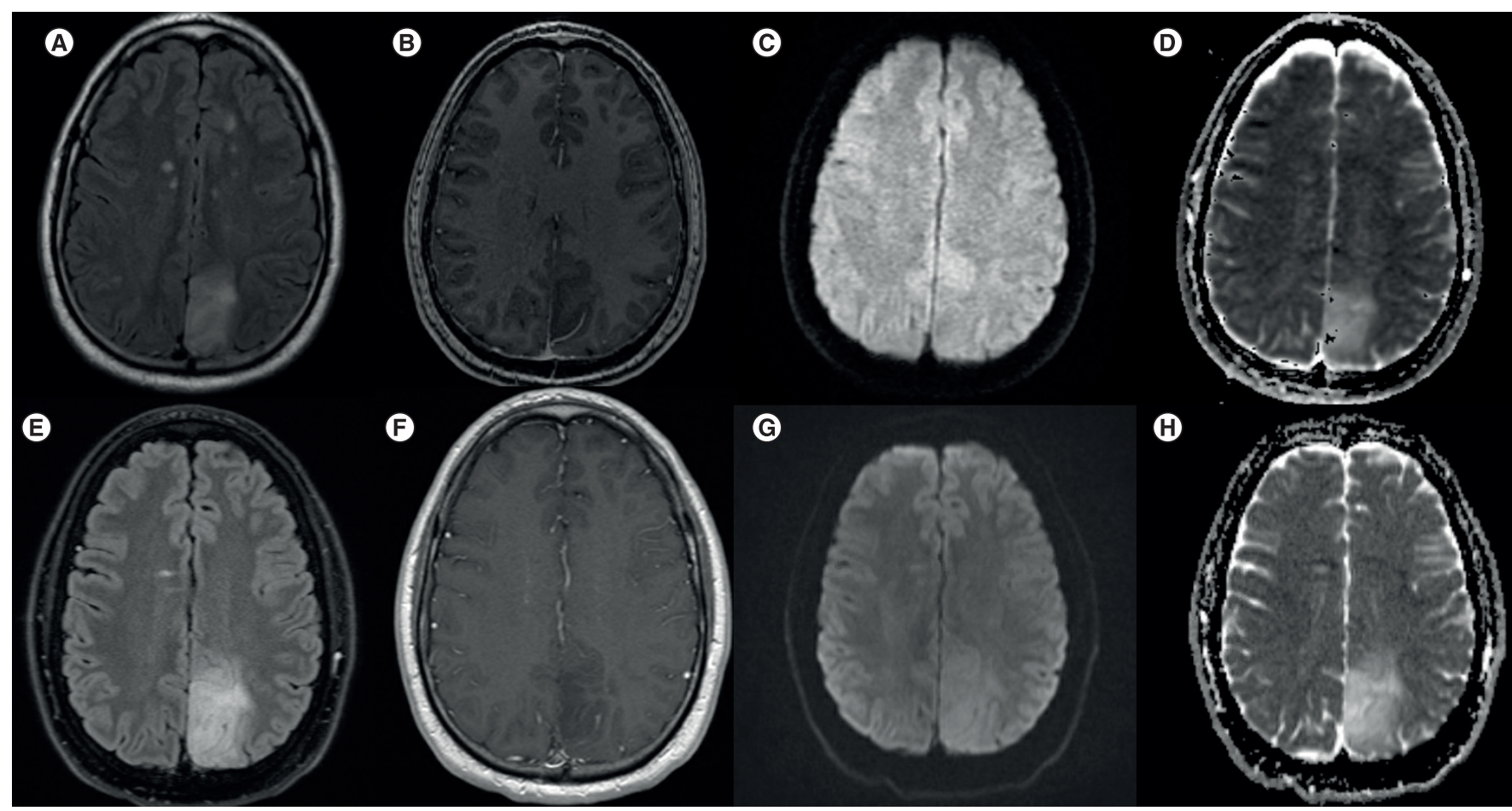

G

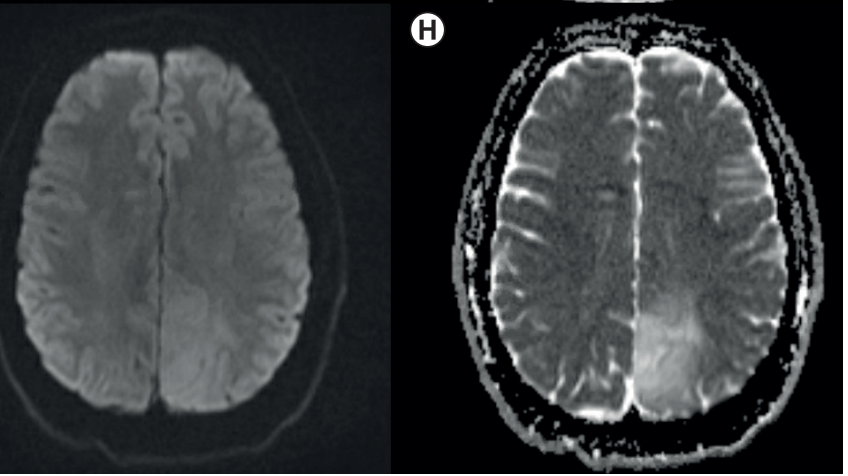

Figure 1. Patient's initial and pre-surgical MRI imaging of the brain with and without contrast. Initial MRI brain imaging: FLAIR (A), T1+ contrast (B), DWI (C) and ADC (D), and at progression/before surgical resection MRI brain imaging: FLAIR (E), T1 + contrast (F), DWI (G) and $A D C(H)$.

ADC: Apparent diffusion coefficient; DWI: Diffusion-weighted imaging; FLAIR: Fluid attenuated inversion recovery.

T2/FLAIR signal within the left parieto-occipital region with associated patchy enhancement (measured $5.9 \times$ $4.9 \times 2.9 \mathrm{~cm}$ ) numerous periventricular white matter lesions and enhancement of lesions within the left centrum semiovale and adjacent to the left temporal horn. Additional testing included lumbar puncture which was notable for eight oligoclonal bands. These findings culminated in a diagnosis of probable MS and hence TD based on FLAIR and T1 contrasted imaging despite findings from diffusion-weighted imaging and apparent diffusion coefficient sequences that were not consistent with TD. Neurology providers initiated the patient on glatiramer $\left(\right.$ Copaxone $\left.{ }^{\circledR}\right)$. Following a second seizure 6 months after presentation, the patient had repeat imaging. The MRI revealed a slight decrease in the size of the left parieto-occipital lesion, but several new lesions were noted in T2 imaging, many of which were enhanced (Figure 1). Due to this progressive imaging, glatiramer was discontinued and the patient was initiated on natalizumab (Tysabri ${ }^{\circledR}$ ). As a selective adhesion molecule inhibitor, natalizuamb prevents $\mathrm{T}$ lymphocytes from penetrating the blood-brain barrier and impeding the demyelination process [12]. Due to the risk of progressive multifocal leukoencephalopathy (PML), prior to initiation of natalizumab therapy patients must have a baseline MRI scan [13]. Certain risk factors place patients at an increased risk of developing PML, including: duration of natalizumab therapy; prior use of immunosuppressants; and the presence of anti-JC virus antibodies. Of significance, our patient was JC virus positive, and providers monitored his viral status based on risk of PML associated with JC virus. Consequently, due to presence of JC virus positivity and risk of PML after 24 months of natalizumab therapy, the patient transitioned to alemtuzumab (Lemtrada ${ }^{\circledR}$ ) therapy. Alemtuzumab is humanized antibody against CD52. Prolonged reduction of $\mathrm{CD} 4^{+}$lymphocyte counts occur with alemtuzumab therapy; infections have been reported in $71 \%$ of alemtuzumab-treated patients and fatal infections have been reported in MS [14]. The MRI following discontinuation of natalizumab was consistent with stable disease with no new or enhancing lesions. However, in the 6 months after initiation of alemtuzumab, the patient continued to have seizures and then developed severe lymphopenia, presumably due to alemtuzumab. Despite years of appropriate therapy for MS review of MRI imaging and progression of the left parieto-occipital lesion prompted a transfer of care and consultation with the neurosurgery team at Duke University Hospital (NC, USA) (Figure 1). The patient underwent biopsy in January 2018 ( $\sim 3 \frac{1}{2}$ years after initial presentation). Final pathology results demonstrated 
an oligodendroglioma (WHO Grade II), 1p/19q codeleted, IDH-1 mutant. In April 2018, he had a gross total resection of the left parieto-occipital tumor. It is important to note that since surgery, the patient has not had any further seizures. Based on the favorable prognostic markers of his tumor and gross total resection, his neuro-oncology providers are following him with serial imaging.

\section{Discussion}

The current case report presents a young male patient with a left parietal oligodendroglioma (WHO Grade II), who was initially misdiagnosed with TD. On MRI scan, oligodendrogliomas often mimic the appearance of TD. Oligodendroglioma is a tumor derived from glial cells involved in myelin production. Oligodendrogliomas are classified by the WHO classification of tumors into oligodendrogliomas (grade II) or anaplastic oligodendrogliomas (grade III) and account for approximately 15-20\% of all gliomas [15]. They are slow-growing tumors that can go undetected for years prior to diagnosis. These tumors are characterized by a codeletion of $1 \mathrm{p} / 19 \mathrm{q}$ and usually have $I D H$ mutations [16]. The most common sites of this tumor are the frontal and temporal lobes and the most common presenting symptoms are seizures [17]. The majority of oligodendroglioma cases present in adults between the third and fifth decades of life.

Some authors have proposed a causal association between oligogendroglioma and MS [18]. Our patient presented with an atypical symptom of MS, a seizure. Of all MS patients, $<2 \%$ are reported to experience a seizure in their lifetime [19].

Disease-modifying agents are a standard of care in the treatment of MS. Long-term exposure to these agents can result in a variety of clinical and financial implications. In addition, the use of glatiramer has been shown to have a possible role in abnormal neurogenesis. This neurogenesis has been linked to the formation of certain primary tumors; however, the effect on existing tumors is not fully understood [20,21]. After clinical and radiographical progression despite appropriate therapy it would have been reasonable to obtain a biopsy sooner in the treatment course to ensure the correct diagnosis [22]. Earlier detection of the oligodendroglioma would have spared this patient from exposure to aggressive therapies. Furthermore, it is unknown what the untoward effects these longterm immunosuppressive agents may have had on his oligodendroglioma.

Although rare, there are documented cases of concurrent oligodendroglioma and MS [23-28]. Radiographic features indicative for glial tumors include mass effect, presence of necrosis and degree of heterogeneity of contrast enhancement. MS lesions are typically found in the periventricular area, spinal cord and optic nerves. Some features including incomplete rim enhancement, absence of mass effect and absence of cortical involvement are more suggestive of TD lesions. In our patient case, the tumefactive lesion is cortical; based on location, it should not have been identified as a TD lesion. Despite diagnostic differences, lack of MRI specificity remains a challenge, especially in the presence of active MS, edema and inflammatory processes [29]. CT-PET imaging can be utilized to identify malignancies but a confirmatory brain biopsy may be required for patients with overlapping features such as atypical ring-enhancing lesions and mass effect $[30,31]$.

\section{Conclusion}

This case report highlights the importance of a brain biopsy in a patient with a brain lesion and the presence of nonspecific radiographic features. An appropriate and timely diagnosis may have prevented exposure to potentially harmful agents as well as progression of symptoms.

\section{Executive summary}

- Brain tumors, including low-grade gliomas, can mimic multiple sclerosis.

- When one diagnoses tumefactive demyelination by imaging, providers should consider other diagnoses such as primary brain tumors.

- If tumefactive demyelination does not respond to escalating therapies, providers should consider alternative diagnoses. 


\section{Ethical conduct of research}

The authors state that they have obtained appropriate institutional review board approval or have followed the principles outlined in the Declaration of Helsinki for all human or animal experimental investigations. In addition, for investigations involving human subjects, informed consent has been obtained from the participants involved.

\section{Informed consent disclosure}

The authors state that they have obtained informed consent from the patient for the inclusion of their medical and treatment history within this case report.

\section{Open access}

This work is licensed under the Attribution-NonCommercial-NoDerivatives 4.0 Unported License. To view a copy of this license, visit http://creativecommons.org/licenses/by-nc-nd/4.0/

\section{References}

Papers of special note have been highlighted as: $\bullet$ of interest

1. Walton C, King R, Rechtman L et al. Rising prevalence of multiple sclerosis worldwide: insights from the Atlas of MS, third edition. Mult. Scler. 26(14), 1816-1821 (2020).

2. Hemond CC, Bakshi R. Magnetic resonance imaging in multiple sclerosis. Cold Spring Harb. Perspect. Med. 8(5), a028969 (2020).

3. Vargas DL, Tyor WR. Update on disease-modifying therapies for multiple sclerosis. J. Investig. Med. 65(5), 883-891 (2017).

4. Straus Farber R, Harel A, Lublin F. Novel agents for relapsing forms of multiple sclerosis. Annu. Rev. Med. 67, 309-321 (2016).

5. Sánchez P, Meca-Lallana V, Barbosa A, Manzanares R, Palmí I, Vivancos J. Tumefactive demyelinating lesions of 15 patients: clinico-radiological features, management and review of the literature. J. Neurol. Sci. 381, 32-38 (2017).

- Focuses on the radiological features of tumefactive demyelination and associated clinical considerations.

6. Patriarca L, Torlone S, Ferrari F, et al. Is size an essential criterion to define tumefactive plaque? MR features and clinical correlation in multiple sclerosis. Neuroradiol. J. 29(5), 384-389 (2016).

7. Frederick MC, Cameron MH. Tumefactive demyelinating lesions in multiple sclerosis and associated disorders. Curr. Neurol. Neurosci. Rep. 16(3), 26 (2016).

8. Altintas A, Petek B, Isik N et al. Clinical and radiological characteristics of tumefactive demyelinating lesions: follow-up study. Mult. Scler. 18(10), 1448-1453 (2012).

9. Lucchinetti CF, Gavrilova RH, Metz I et al. Clinical and radiographic spectrum of pathologically confirmed tumefactive multiple sclerosis. Brain 131(7), 1759-1775 (2008).

10. Qi W, Jia GE, Wang X, Zhang M, Ma Z. Cerebral tumefactive demyelinating lesions. Oncol. Lett. 10(3), 1763-1768 (2015).

11. Kilic AK, Kurne AT, Oguz KK, Soylemezoglu F, Karabudak R. Mass lesions in the brain: tumor or multiple sclerosis? Clinical and imaging characteristics and course from a single reference center. Turk. Neurosurg. 23(6), 728-735 (2013).

12. Tysabri (natalizumab) [prescribing information]. Biogen Inc, MA, USA (2018). https://www.accessdata.fda.gov/drugsatfda_docs/label/2012/125104s0576lbl.pdf

13. US Food and Drug Administration. Tysabri (natalizumab) intravenous injection monoclonal antibody. Risk evaluation and mitigation strategy. (2012). http://www.fda.gov/downloads/Drugs/DrugSafety/PostmarketDrugSafetyInformationforPatientsandProviders/UCM288126.pdf

14. Adang L, Berger J. Progressive multifocal leukoencephalopathy. F1000Res. 4(F1000 Faculty Rev), 1424 (2015).

15. DeAngelis LM, Wen PY. Primary and metastatic tumors of the nervous system. In: Harrison's Principles of Internal Medicine 20th Edition. Jameson J, Fauci AS, Kasper DL, Hauser SL, Longo DL, Loscalzo J. (Eds). McGraw-Hill, NY, USA, (2018).

16. Jenkins RB, Blair H, Ballman KV et al. A t $(1 ; 19)(\mathrm{q} 10 ; \mathrm{p} 10)$ mediates the combined deletions of $1 \mathrm{p}$ and $19 \mathrm{q}$ and predicts a better prognosis of patients with oligodendroglioma. Cancer Res. 66(20), 9852-9861 (2006).

17. Lassman AB, Iwamoto FM, Cloughesy TF et al. International retrospective study of over 1000 adults with anaplastic oligodendroglial tumors. Neuro. Oncol. 13(6), 649-659 (2011).

18. Carvalho AT, Linhares P, Castra L, Sá MJ. Multiple sclerosis and oligodendroglioma: an exceptional association. Case Rep. Neurol. Med. 2014, 546817 (2014).

19. Sponsler JL, Kendrick-Adey AC. Seizures as a manifestation of multiple sclerosis. Epileptic Disord. 13(4), 401-410 (2011).

- Points out that seizures can occur in patients with multiple sclerosis.

20. Batista CM, Mariano ED, Barbosa BJ et al. Adult neurogenesis and glial oncogenesis: when the process fails. Biomed Res. Int. 2014, 438639 (2014). 
21. Carvalho AT, Linhares P, Castra L, Sá MJ. Multiple sclerosis and oligodendroglioma: an exceptional association. Case Rep. Neurol. Med. 2014, 546817 (2014).

22. Hardy TA, Chataway J. Tumefactive demyelination: an approach to diagnosis and management. J. Neurol. Neurosurg. Psychiatry 84(9), 1047-1053 (2013).

23. Green AJ, Bollen AW, Berger MS, Oksenberg JR, Hauser SL. Multiple sclerosis and oligodendroglioma. Mult. Scler. 7(4), 269-273 (2001).

24. Carvalho AT, Linhares P, Castra L, Sá MJ. Multiple sclerosis and oligodendroglioma: an exceptional association. Case Rep. Neurol. Med. 2014, 546817 (2014).

- Report is a modern discussion of a case of multiple sclerosis with oligodendroglioma.

25. Khan OA, Bauserman SC, Rothman MI, Aldrich EF, Panitch HS. Concurrence of multiple sclerosis and brain tumor: clinical considerations. Neurology 48(5), 1330-1333 (1997).

26. Shankar SK, Rao TV, Srivastav VK, Narula S, Asha T, Das S. Balo’s concentric sclerosis: a variant of multiple sclerosis associated with oligodendroglioma. Neurosurgery 25(6), 982-986 (1989).

27. Gordana MT, Mauro A, Soffietti R, Leone M. Association between multiple sclerosis and oligodendroglioma. Case report. Ital. J. Neurol. Sci. 2(4), 403-409 (1981).

28. Šega S, Horvat A, Popovic M. Anaplastic oligodendroglioma and gliomatosis type 2 in interferon- $\beta$ treated multiple sclerosis patients: report of two cases. Clin. Neurol. Neurosurg. 108(3), 259-265 (2006).

29. Sinclair G, Al-saffar Y, Johnstone P, Hatiboglu MA, Shamikh A. A challenging case of concurrent multiple sclerosis and anaplastic astrocytoma. Surg. Neurol. Int. 10, 166 (2019).

30. Hardy TA. Pseudotumoral demyelinating lesions: diagnostic approach and long-term outcome. Curr. Opin. Neurol. 32(3), 467-474 (2019).

31. Sinha MK, Garg RK, Bhatt MLB, Chandra A. Tumefactive demyelinating lesion: experience with two unusual patients. J. Postgrad. Med. 56(2), 146-149 (2010). 